\title{
Feedback autoregulation of gene expression always amplifies noise in timing of threshold crossings
}

\author{
Zahra Vahdat, Khem Raj Ghusinga and Abhyudai Singh ${ }^{1}$
}

\begin{abstract}
Many cellular events occur when their corresponding regulatory proteins attain critical thresholds. Can cells schedule such events with precision by controlling the dynamics of the proteins? We investigate this question by considering a simple gene expression model that consists of switching the gene between $O F F$ and $O N$ states and degradation of the protein. Because feedback regulation is a pervasive method of control in biological systems, we analyzed three feedback mechanisms (protein regulates either its own transcription, or the rate at which gene turns $O N$, or the rate at which gene turns OFF) for their abilities to suppress noise in timing. We show that in the limiting case where the protein does not degrade, feedbacks always amplify noise in event timing.
\end{abstract}

\section{INTRODUCTION}

Cells use timekeeping apparatuses for various purposes such as circadian rhythms, cell-fate decisions, coordination of responses to external stimuli, etc. [1]-[6]. Perhaps the most straightforward mechanism to govern a cellular event's timing is to couple it with a regulatory protein crossing a critical threshold level [1]. Some examples where such mechanisms occur are, among others, lysis of an E. coli cell infected by bacteriophage $\lambda$, passage of a mammalian cell through restriction point, meiosis, apoptosis, and development [7]-[17]. These timers consist of biochemical species whose dynamics are inherently stochastic [18]-[23]. How then cells control these biochemical species' dynamics to ensure precision in timing is a fundamental question of interest.

Recent works have modeled event timing as a firstpassage time problem (FPT) [24], [25], particularly in gene expression models [26]-[34]. The question of precision in event timing is investigated by searching the parameter space to find parameters that minimize noise (quantified by the coefficient of variation) for a constant mean and exploring whether feedback regulation suppresses noise. These works show an optimal threshold (approximately half of the steadystate protein level) that minimizes noise in event timing [26], [31], [32], [35]. Another key takeaway is that for a stable protein, any form of feedback gives higher noise in event timing around a fixed mean time [32] and that this result is robust to physiologically relevant variations in model parameters [32], [36] and extrinsic disturbances [37]. However, incorporating protein degradation requires positive feedback to transcription rate to suppress noise in event timing [32]. It is also worth pointing out that

\footnotetext{
1 Department of Electrical and Computer Engineering, Biomedical Engineering, Mathematical Sciences, Center for Bioinformatics and Computational Biology, University of Delaware, Newark, DE USA 19716 absingh@udel . edu
}

the optimal feedback strategy to ensure precision in event timing depends upon model assumptions [33] as well as constraints [35] while comparing the feedback strategies. Here we examine the optimal feedback strategy to minimize the noise in event timing for a gene expression model in which the promoter switches between OFF and ON states. We specifically consider feedback mechanisms that operate on one of the gene switching rates or the transcription rate. For the particular case when the protein is stable, our analysis shows that any form of feedback always increases noise in timing.

\section{MODEL FORMULATION}

Let $x(t)$ denote the level of the timekeeper protein at time $t$. We are interested in the first-passage time (FPT), denoted by $T$, for $x(t)$ to cross a threshold $X$ for the first time:

$$
T:=\inf \{t: x(t) \geq X\} .
$$

To describe the protein dynamics, we consider a gene expression model shown in Fig. 1 (a). In this model, the gene transitions between two states, OFF and ON [20], [22], [38][44]. Protein production occurs when the gene is in the ON state. We assume that to achieve precision in the event timing, one of the following feedback mechanisms may be employed:

I - The production rate of the protein depends on the protein level.

II - The rate at which the gene switches to the ON state depends on the protein level,

III - The rate at which the gene switches to the OFF state depends on the protein level.

We consider the rate parameters as $u, v$, and $k$ when there is no feedback regulation. We denote these feedback mechanisms using a subscript $x$ on the rate parameters. Thus, $u_{x}, v_{x}$ and $k_{x}$ denote the rate at which the gene turns $\mathrm{ON}$, the rate at which the gene turns OFF and the transcription rate respectively, when $x(t)$ is the protein level at time $t$. We also assume gene expression in translation burst limit, where each production event produces a burst of protein molecules [36], [45]. Often, the burst size distribution is geometric [32], but here we make the simplifying assumption that it is drawn from a degenerate distribution such that burst size is one with probability one. Finally, we assume that protein degradation occurs at a rate of $\gamma$.

Our goal is to compute the pdf of FPT defined in (1). To that end, one needs to characterize the time evolution of the stochastic process $x(t)$ until it crosses the threshold $X$ (Fig. 1 (b)). Let $p_{0, x}$ be the probability that the gene is in the 



Time

Fig. 1. Event timing for a model of gene expression with feedback regulation. (a) The gene switches between OFF and ON states. Protein production occurs when the gene is in the ON state. The feedbacks (dashed lines) imply that the transition rates depend upon the protein level $x(t)$, shown by the subscript $x$. The rates are shown next to the corresponding transitions. (b) Event timing is modeled as the first-passage time for the protein level to hit a threshold. Each trajectory corresponds to a realization of the stochastic process $x(t)$.

OFF state and $x(t) \in\{0,1, \ldots, X-1\}$. Likewise, let $p_{1, x}$ be the probability that the gene is in the ON state. Collecting the probabilities of all states until $x(t)$ crosses $X$

$$
\boldsymbol{P}(t):=\left[\begin{array}{llllll}
p_{0,0} & \cdots & p_{0, X-1} & p_{1,0} & \cdots & p_{1, X-1}
\end{array}\right]^{\top},
$$

we compute the pdf of $T, f_{T}(t)$, as [32], [37]

$$
f_{T}(t)=k_{X-1} p_{1, X-1}(t)
$$

Here, the superscript ${ }^{\top}$ denotes transpose of a vector/matrix. The expression in (3) may be written as

$$
f_{T}(t)=\boldsymbol{U}^{\top} \boldsymbol{P}(t)
$$

where $U$ is the vector

$$
\boldsymbol{U}=\left[\begin{array}{llll}
0 & \ldots & 0 & k_{X-1}
\end{array}\right]^{\top} .
$$

Finally, $\boldsymbol{P}(t)$ is governed by the forward Kolmogorov equation (also known as the Chemical Master Equation)

$$
\frac{d \boldsymbol{P}(t)}{d t}=\boldsymbol{A P}(t)
$$

using which yields for $f_{T}(t)$

$$
f_{T}(t)=\boldsymbol{U}^{\top} \exp (\boldsymbol{A} t) \boldsymbol{P}(0) .
$$

Here the vector $\boldsymbol{P}(0)$ is the initial probability distribution. We assume that at $t=0$ the gene in the ON state and $x(0)=$ 0 , resulting in

$$
\boldsymbol{P}(0)=\left[\begin{array}{lllllll}
0 & \ldots & 0 & 1 & 0 & \ldots & 0
\end{array}\right]^{\top} .
$$

The matrix $\boldsymbol{A}$ is block-diagonal and is given by

$$
\boldsymbol{A}=\left[\begin{array}{ll}
\boldsymbol{A}_{00} & \boldsymbol{A}_{01} \\
\boldsymbol{A}_{10} & \boldsymbol{A}_{11}
\end{array}\right],
$$

where $(m, n)$-th elements of the sub-matrices are as follows

$$
\begin{aligned}
& \left(\boldsymbol{A}_{00}\right)_{m n}= \begin{cases}m \gamma & n=m+1 \\
-u_{m-1}-(m-1) \gamma & n=m \\
0 & \text { otherwise }\end{cases} \\
& \left(\boldsymbol{A}_{01}\right)_{m n}= \begin{cases}v_{m-1} & n=m \\
0 & \text { otherwise }\end{cases} \\
& \left(\boldsymbol{A}_{10}\right)_{m n}= \begin{cases}u_{m-1} & n=m \\
0 & \text { otherwise }\end{cases} \\
& \left(\boldsymbol{A}_{11}\right)_{m n}= \begin{cases}0 & n>m+1 \\
m \gamma & n=m+1 \\
-v_{m-1}-k_{m-1} & n=m \\
-(m-1) \gamma & n=m-1 \\
k_{m-2} & n\end{cases}
\end{aligned}
$$

with $m, n \in\{1, \ldots, X\}$.

To compute the optimal feedback strategy that minimizes noise in timing, we require the statistical moments (mean and variance) of $T$. In what follows, we provide semi-analytical formulas for the moments and provide exact formulas for the particular case when the protein does not degrade.

\section{EXACT STATISTICAL MOMENTS OF FPT}

We compute the moments of $T$ using its pdf from (7). It turns out that $\boldsymbol{A}$ is a Hurwitz stable matrix as it has negative diagonal elements and, the sum of absolute values of nondiagonal elements for each column is less than the absolute value of the diagonal element [37]. The mean and the secondorder moment of FPT are given by

$$
\langle T\rangle=\boldsymbol{U}^{\top} \boldsymbol{A}^{-2} \boldsymbol{P}(0), \quad\left\langle T^{2}\right\rangle=-2 \boldsymbol{U}^{\top} \boldsymbol{A}^{-3} \boldsymbol{P}(0),
$$

where $\boldsymbol{A}^{-1}$ is inverse of matrix $\boldsymbol{A}, \boldsymbol{U}^{\top}$ is transpose of vector $\boldsymbol{U}$ in (5), and $\boldsymbol{P}(0)$ is in (8). These formulas can be easily computed numerically, and they simplify considerably in the particular case of $\gamma=0$ (stable protein) as we show below.

\section{A. Moments of FPT when $\gamma=0$}

Because the matrix $\boldsymbol{A}$ in (9a) is a block diagonal, its inverse also has a block-diagonal form [46]

$$
\boldsymbol{A}^{-1}=\left[\begin{array}{ll}
\boldsymbol{A}_{00}^{\prime} & \boldsymbol{A}_{01}^{\prime} \\
\boldsymbol{A}_{10}^{\prime} & \boldsymbol{A}_{11}^{\prime}
\end{array}\right] \text {. }
$$

The exact forms of $\boldsymbol{A}_{00}^{\prime}, \boldsymbol{A}_{01}^{\prime}, \boldsymbol{A}_{10}^{\prime}$ and $\boldsymbol{A}_{11}^{\prime}$ may be computed using standard linear algebra. It is possible to obtain analytical forms of these matrices when $\gamma=0$ (see 
the Appendix). Using these analytical forms, it is easy to show that the term $\boldsymbol{U}^{\top} \boldsymbol{A}^{-1}$ in (10) simplifies to [37]

$$
\boldsymbol{U}^{\top} \boldsymbol{A}^{-1}=-\left[\begin{array}{llll}
1 & 1 & \ldots & 1
\end{array}\right] .
$$

Moreover, when $\gamma=0, \boldsymbol{A}^{-1} \boldsymbol{P}(0)$ follows

$$
\boldsymbol{A}^{-1} \boldsymbol{P}(0)=-\left[\begin{array}{llllll}
\frac{v_{0}}{u_{0}} \frac{1}{k_{0}} & \ldots & \frac{v_{X-1}}{u_{X-1}} \frac{1}{k_{X-1}} & \frac{1}{k_{0}} & \ldots & \frac{1}{k_{X-1}}
\end{array}\right]^{\top} .
$$

Finally, simplifying $\boldsymbol{A}^{-2} \boldsymbol{P}(0)$ gives

$$
\begin{aligned}
\boldsymbol{A}^{-2} \boldsymbol{P}(0) & =-\left[\begin{array}{ll}
\boldsymbol{A}_{00}^{\prime} & \boldsymbol{A}_{01}^{\prime} \\
\boldsymbol{A}_{10}^{\prime} & \boldsymbol{A}_{11}^{\prime}
\end{array}\right]\left[\begin{array}{c}
\boldsymbol{\zeta} \boldsymbol{c} \\
\boldsymbol{\kappa}
\end{array}\right] \\
& =-\left[\begin{array}{cc}
\boldsymbol{\zeta} \boldsymbol{A}_{11}^{\prime} & \boldsymbol{\zeta} \boldsymbol{A}_{11}^{\prime} \\
\boldsymbol{A}_{11}^{\prime} & \boldsymbol{A}_{11}^{\prime}
\end{array}\right]\left[\begin{array}{c}
\boldsymbol{\zeta} \boldsymbol{\kappa} \\
\boldsymbol{\kappa}
\end{array}\right]-\left[\begin{array}{c}
\boldsymbol{\eta} \boldsymbol{\zeta} \boldsymbol{c} \\
0
\end{array}\right] \\
& =-\left[\begin{array}{c}
\boldsymbol{\zeta} \boldsymbol{A}_{11}^{\prime}(\boldsymbol{\zeta}+I) \boldsymbol{\kappa} \\
\boldsymbol{A}_{11}^{\prime}(\boldsymbol{\zeta}+I) \boldsymbol{\kappa}
\end{array}\right]-\left[\begin{array}{c}
\boldsymbol{\eta} \boldsymbol{\zeta} \boldsymbol{\kappa} \\
0
\end{array}\right],
\end{aligned}
$$

where the vector $\boldsymbol{\kappa}$ and the matrices $\zeta$ and $\boldsymbol{\eta}$ are given by

$$
\begin{aligned}
\boldsymbol{\kappa} & =\left[\begin{array}{lll}
\frac{1}{k_{0}} & \cdots & \frac{1}{k_{X-1}}
\end{array}\right]^{\top}, \\
\boldsymbol{\zeta} & =\operatorname{diag}\left[\begin{array}{lll}
\frac{v_{0}}{u_{0}} & \cdots & \frac{v_{X-1}}{u_{X-1}}
\end{array}\right], \\
\boldsymbol{\eta} & =\operatorname{diag}\left[\begin{array}{lll}
\frac{-1}{u_{0}} & \cdots & \frac{-1}{u_{X-1}}
\end{array}\right] .
\end{aligned}
$$

Using the above results, the first two moments of FPT when $\gamma=0$ simplify to

$$
\begin{aligned}
\langle T\rangle= & \sum_{x=0}^{X-1} \frac{1}{k_{x}}\left(1+\frac{v_{x}}{u_{x}}\right), \\
\left\langle T^{2}\right\rangle= & \left(\sum_{x=0}^{X-1} \frac{1}{k_{x}}\left(1+\frac{v_{x}}{u_{x}}\right)\right)^{2} \\
& +\sum_{x=0}^{X-1} \frac{1}{k_{x}^{2}}\left(1+\frac{v_{x}}{u_{x}}\right)^{2}+\sum_{x=0}^{X-1} \frac{2}{k_{x}} \frac{v_{x}}{u_{x}^{2}} .
\end{aligned}
$$

Throughout the paper we use the mean FPT $\langle T\rangle$ and FPT noise quantified by the squared coefficient of variation

$$
C V_{T}^{2}=\frac{\left\langle T^{2}\right\rangle-\langle T\rangle^{2}}{\langle T\rangle^{2}}
$$

The formulas in (15) provide insights into how the FPT statistics depend upon the model parameters. For example, if we consider the case of no feedback $\left(k_{x}=k, u_{x}=u\right.$, and $v_{x}=v$ ), then we have that

$$
\begin{aligned}
\langle T\rangle & =\frac{X}{k} \frac{u+v}{u}, \\
C V_{T}^{2}=\frac{\left\langle T^{2}\right\rangle-\langle T\rangle^{2}}{\langle T\rangle^{2}} & =\frac{1}{X}+\frac{2}{\langle T\rangle} \frac{v}{u(u+v)} .
\end{aligned}
$$

Here $1 / X$ is the Poisson limit of the noise if there is no gene switching $(v \rightarrow 0)$. The second term is the contribution of gene switching to the overall noise, depending upon $u$ and $v$. As is clear from the expressions in (15), feedback mechanisms would also affect the moments. We defer the discussion on this to section IV.

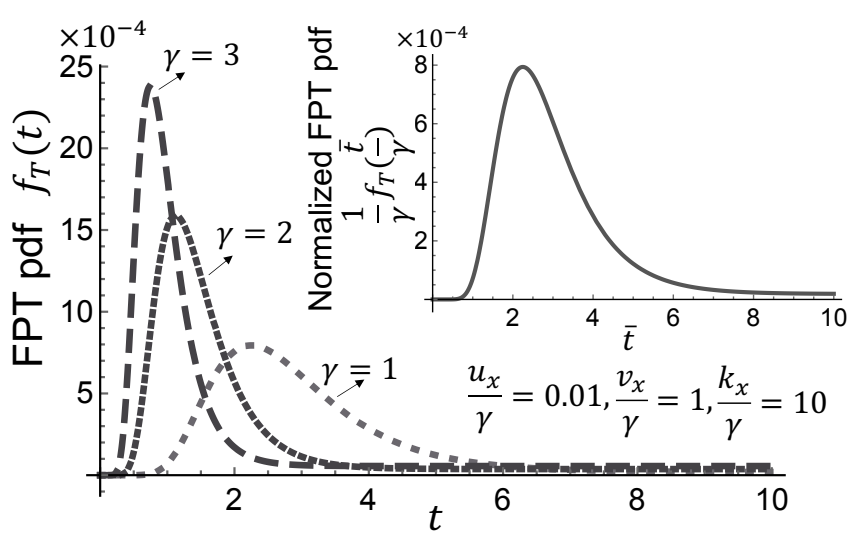

Fig. 2. First-passage time distribution is scale-invariant with respect to the protein degradation rate. Left: FPT probability distribution when $\gamma \neq 0$. When transcription rate $k_{x}$ and gene switching rates $u_{x}$ and $v_{x}$ scale by $\gamma$, the FPT and its pdf also scale by $\gamma$. Right: Upon normalizing FPT by protein dilution rate $\gamma$, the distributions collapse upon each other.

\section{B. Moments when $\gamma \neq 0$}

When the degradation of protein is considered $(\gamma \neq 0)$, it is difficult to obtain closed-form expressions for FPT moments as those in (15); so, we work with the semianalytical form in (10). We find that the moments have a scale-invariant property when $\gamma \neq 0$. To see this, we recall the pdf of FPT from (7)

$$
f_{T}(t)=\boldsymbol{U}^{\top} \boldsymbol{P}(t)=\left[\begin{array}{llll}
0 & \ldots & 0 & k_{X-1}
\end{array}\right]^{\top} \exp (\boldsymbol{A} t) \boldsymbol{P}(0) .
$$

Let $\bar{T}=\gamma T$. The pdf of $\bar{T}$ then follows

$$
f_{\bar{T}}(\bar{t})=\frac{1}{\gamma} f_{T}\left(\frac{\bar{t}}{\gamma}\right)=\frac{\boldsymbol{U}^{\top}}{\gamma} \exp \left(\frac{\boldsymbol{A}_{\bar{t}}}{\gamma}\right) \boldsymbol{P}(0) .
$$

Thus, if we scale the rate parameters $u_{x}, v_{x}$, and $k_{x}$ by $\gamma$, then the above pdf does not depend upon the specific value of $\gamma$ but only at the ratios $u_{x} / \gamma, v_{x} / \gamma$, and $k_{x} / \gamma$ (Fig. 2).

A consequence of the scale-invariant property is that the moments of $T$ and $\bar{T}=\gamma T$ have the following relationships:

$$
\langle\bar{T}\rangle=\gamma\langle T\rangle, \quad \frac{\left\langle\bar{T}^{j}\right\rangle}{\langle\bar{T}\rangle^{j}}=\frac{\left\langle T^{j}\right\rangle}{\langle T\rangle^{j}} \forall j \geq 2 .
$$

With this property in mind, we set $\gamma=1$ which implies that $u_{x}, v_{x}, k_{x}$, and $\langle T\rangle$ are scaled by $\gamma$. How do the FPT moments depend upon the scaled model parameters when $\gamma \neq 0$ ? As done in section III-A, we analyze the no feedback case here.

We first examine the simple case of constitutive gene expression (i.e., the gene is always $\mathrm{ON}$ ). In this case, we find that $\langle T\rangle$ and $C V_{T}^{2}$ are decreasing functions of $k / \gamma$. Incidentally, $k / \gamma$ is the mean of the steady-state distribution of $x(t)$, which we refer to as $x_{s s}$. In the limiting case when $x_{s s} \gg X$, we find that $C V_{T}^{2}$ approaches the Poisson limit of $1 / X$. Next, we investigate how the introduction of gene switching influences the behaviors of FPT moments. It may be seen that in this case

$$
x_{s s}=\frac{k}{\gamma} \frac{\frac{u}{\gamma}}{\gamma}+\frac{v}{\gamma}=\frac{k}{\gamma} \frac{u}{u+v} .
$$


We find that the moments depend both on $u$ and $v$, but the qualitative trend of $\langle T\rangle$ remains similar in the sense that it decreases with an increase in $x_{s s}$. However, the qualitative behavior of $C V_{T}^{2}$ alters, except for the case when gene switching occurs much faster than the timescale set by $\gamma$. Notably, $C V_{T}^{2}$ exhibits non-monotonic behavior in some cases, while in other cases, it only shows an increasing trend with $x_{s s}$ as opposed to the decreasing trend shown in the absence of gene switching (Fig. 3).

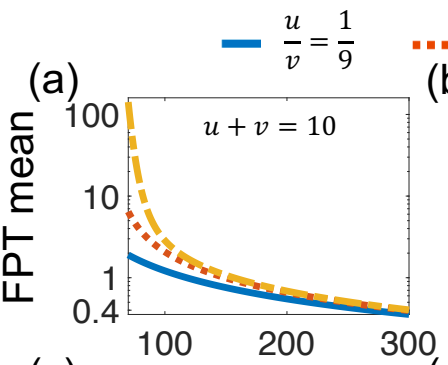

(c) (d)

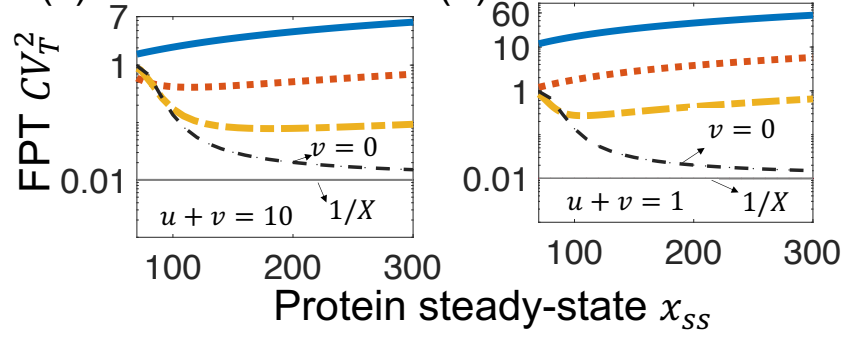

Fig. 3. FPT moments with variations in steady-state protein level through different parameters for the case that there is no feedback regulation. The mean FPT always decreases as the steady-state protein level increases. However, FPT noise may increase, decrease, or show nonmonotonic behavior with the increase in mean steady-state. The parameter values are as shown in the plot. The threshold is taken to be $X=100$.

\section{EFFECT OF FEEDBACK ON FPT NOISE}

This section examines the effect of feedback regulation on FPT statistics and investigates the optimal feedback strategy that minimizes noise in timing around a given fixed mean FPT. Because $\langle T\rangle$ is constant, minimizing $C V_{T}^{2}$ is same as minimizing $\left\langle T^{2}\right\rangle$. Thus, we solve the following equivalent optimization problem [32]:

$$
\min _{k_{x}, u_{x}, v_{x}}\left\langle T^{2}\right\rangle+\lambda(\langle T\rangle-c), \quad \forall x \in 0 \ldots, X-1 .
$$

In the above setup, $\lambda$ is a regularization parameter akin to the Lagrange multiplier, and $c$ is the fixed value of mean event timing. We provide analytical solutions to the optimal feedback strategy for the case of $\gamma=0$ and solve the above optimization problem numerically when $\gamma \neq 0$. We only need to analyze the case of $\gamma=1$ as facilitated by the scaleinvariance property.

To find the optimal feedback for stable protein $(\gamma=0)$, we use the moments from (15) in the cost function in (22).
The optimal solution must satisfy

$$
\begin{aligned}
& \frac{\partial\left\langle T^{2}\right\rangle}{\partial k_{x}}+\lambda \frac{\partial\langle T\rangle}{\partial k_{x}}=0, \\
& \frac{\partial\left\langle T^{2}\right\rangle}{\partial u_{x}}+\lambda \frac{\partial\langle T\rangle}{\partial u_{x}}=0, \\
& \frac{\partial\left\langle T^{2}\right\rangle}{\partial v_{x}}+\lambda \frac{\partial\langle T\rangle}{\partial v_{x}}=0, \\
& \langle T\rangle-c=0 .
\end{aligned}
$$

Taking derivative of $\langle T\rangle$ and $\left\langle T^{2}\right\rangle$ in (15) w.r.t $k_{x}, u_{x}$ and $v_{x}$ for $0 \leq x \leq X-1$ yields

$$
\begin{aligned}
& \frac{\partial\langle T\rangle}{\partial k_{x}}=-\left(1+\frac{v_{x}}{u_{x}}\right) \frac{1}{k_{x}^{2}}, \\
& \frac{\partial\langle T\rangle}{\partial u_{x}}=-\frac{v_{x}}{k_{x} u_{x}^{2}} \\
& \frac{\partial\langle T\rangle}{\partial v_{x}}=\frac{1}{k_{x} u_{x}}
\end{aligned}
$$

$$
\begin{aligned}
& \frac{\partial\left\langle T^{2}\right\rangle}{\partial k_{x}}=-\frac{2 v_{x}}{u_{x}^{2}} \frac{1}{k_{x}^{2}}-\frac{2}{k_{x}^{2}}\left(1+\frac{v_{x}}{u_{x}}\right) F, \\
& \frac{\partial\left\langle T^{2}\right\rangle}{\partial u_{x}}=-\frac{4 v_{x}}{u_{x}^{3}} \frac{1}{k_{x}}-2 \frac{v_{x}}{k_{x} u_{x}^{2}} F, \\
& \frac{\partial\left\langle T^{2}\right\rangle}{\partial v_{x}}=\frac{2}{u_{x}^{2}} \frac{1}{k_{x}}+\frac{2}{k_{x} u_{x}} F \\
& F=\frac{1}{k_{x}}\left(1+\frac{v_{x}}{u_{x}}\right)+\sum_{l=0}^{X-1} \frac{1}{k_{l}}\left(1+\frac{v_{l}}{u_{l}}\right) .
\end{aligned}
$$

We now consider the three specific feedback strategies as shown in Fig. 4:

I Only production rate is regulated by protein level feedback. Thus, we have $u_{x}=u$ and $v_{x}=v$.

II Only the switching rate from OFF to $\mathrm{ON}$ is regulated by protein level feedback, i.e., $k_{x}=k$ and $v_{x}=v$.

III Only the switching rate from ON to OFF is regulated by protein level feedback, implying $k_{x}=k$ and $u_{x}=$ $u$.

The exact forms of the feedback strategies for the above cases and the minimum achievable FPT noise are tabulated in Fig. 4. Importantly, we find that the best strategy to minimize the noise in timing is to have no feedback at all.

We recall that the optimal feedback strategy for the first scenario above was computed in [37]. Moreover, the limiting case $v \rightarrow 0$ reduces to the gene expression model without gene switching, as studied in [32]. Indeed, our result also reduces to the same optimal feedback strategy

$$
k_{x}^{*}=X / c,
$$

which gives the minimum achievable noise $C V_{T}^{2}=1 / X$.

\section{CONClusion}

How cells ensure precision in their timekeeping apparatuses is a significant fundamental problem. Here we considered a gene expression model with three possible feedback architectures and investigated the best feedback mechanism 


\begin{tabular}{|c|c|c|c|}
\hline \multirow[b]{2}{*}{ Model } & 1 & II & \multirow{2}{*}{ OFF $\underset{v_{x}}{\stackrel{u}{\longrightarrow}}$ ON $\stackrel{k}{\longrightarrow}$ Protein } \\
\hline & 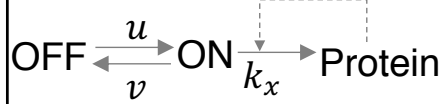 & OFF $\underset{v}{\stackrel{u_{\downarrow}}{\longrightarrow}}$ ON $\stackrel{k}{\longrightarrow}$ Protein & \\
\hline Optimal rate & $k_{x}^{*}=\left(1+\frac{v}{u}\right) \frac{X}{c}$ & $u_{x}^{*}=\frac{v X}{c k-X}$ & $v_{x}^{*}=u\left(\frac{c k}{X}-1\right)$ \\
\hline $\begin{array}{l}\text { Optimal noise } \\
\left(C V_{T}^{2}\right)\end{array}$ & $C V_{T}^{2}=\frac{2}{c u\left(\frac{u}{v}+1\right)}+\frac{1}{X}$ & $C V_{T}^{2}=\frac{2 X}{c^{2} k v}-\frac{4}{c v}+\frac{2 k}{v X}+\frac{1}{X}$ & $C V_{T}^{2}=-\frac{2 X}{c^{2} k u}+\frac{2}{c u}+\frac{1}{X}$ \\
\hline
\end{tabular}

Fig. 4. Optimal feedback strategies and corresponding minimum noise in timing for stable protein. For each feedback strategy $(I, I I$, and $I I I)$, a no feedback regulation minimizes the noise in timing. The corresponding minimum noise is always more significant than the Poisson limit $1 / X$ and depends upon the gene-switching rates. For the strategies $I I$ and $I I I$ the condition $c k>X$ should satisfy to have the optimal solution.

to minimize the noise in events' timing. We found if the timekeeper protein does not degrade, feedbacks always amplify noise in event timing. In future work we investigate the effect of concurrent feedback mechanisms where more than one feedback operates simultaneously.

\section{ACKNOWLEDGMENT}

This work is supported by grants from the Army Research Office (W911NF1910243) and the National Science Foundation (ECCS-1711548).

\section{APPENDIX}

Computation of $\boldsymbol{A}^{-1}$ when $\gamma=0$ :

For the block-diagonal matrix $\boldsymbol{A}, \boldsymbol{A}^{-1}$ has the form given by (11), where

$$
\begin{aligned}
& \boldsymbol{A}_{00}^{\prime}=\boldsymbol{A}_{00}^{-1}+\boldsymbol{A}_{00}^{-1} \boldsymbol{A}_{01}\left(\boldsymbol{A}_{11}-\boldsymbol{A}_{10} \boldsymbol{A}_{00}^{-1} \boldsymbol{A}_{01}\right)^{-1} \boldsymbol{A}_{10} \boldsymbol{A}_{00}^{-1}, \\
& \boldsymbol{A}_{01}^{\prime}=-\boldsymbol{A}_{00}^{-1} \boldsymbol{A}_{01}\left(\boldsymbol{A}_{11}-\boldsymbol{A}_{10} \boldsymbol{A}_{00}^{-1} \boldsymbol{A}_{01}\right)^{-1}, \\
& \boldsymbol{A}_{10}^{\prime}=-\left(\boldsymbol{A}_{11}-\boldsymbol{A}_{10} \boldsymbol{A}_{00}^{-1} \boldsymbol{A}_{01}\right)^{-1} \boldsymbol{A}_{10} \boldsymbol{A}_{00}^{-1}, \\
& \boldsymbol{A}_{11}^{\prime}=\left(\boldsymbol{A}_{11}-\boldsymbol{A}_{10} \boldsymbol{A}_{00}^{-1} \boldsymbol{A}_{01}\right)^{-1} .
\end{aligned}
$$

Using (9b)-(9e) and (11)

$$
\begin{aligned}
& \boldsymbol{A}_{11}^{\prime}=-\left[\begin{array}{cccc}
\frac{1}{k_{0}} & & & \\
\frac{1}{k_{1}} & \frac{1}{k_{1}} & & \\
\frac{1}{k_{2}} & \frac{1}{k_{2}} & \frac{1}{k_{2}} & \\
\vdots & \vdots & \vdots & \\
\frac{1}{k_{X-1}} & \frac{1}{k_{X-1}} & \frac{1}{k_{X-1}} & \frac{1}{k_{X-1}}
\end{array}\right] \\
& \boldsymbol{A}_{10}^{\prime}=\boldsymbol{A}_{11}^{\prime}, \\
& \boldsymbol{A}_{01}^{\prime}=\operatorname{diag}\left[\begin{array}{llll}
\frac{v_{0}}{u_{0}} & \frac{v_{1}}{u_{1}} & \frac{v_{2}}{u_{2}} \ldots & \frac{v_{X-1}}{u_{X-1}}
\end{array}\right] \cdot \boldsymbol{A}_{11}^{\prime} \\
& =-\left[\begin{array}{ccc}
\frac{v_{0}}{u_{0}} \frac{1}{k_{0}} & & \\
\frac{v_{1}}{u_{1}} \frac{1}{k_{1}} & \frac{v_{1}}{u_{1}} \frac{1}{k_{1}} & \\
\vdots & \vdots & \\
\frac{v_{X-1}}{u_{X-1}} \frac{1}{k_{X-1}} & \cdots & \frac{v_{X-1}}{u_{X-1}} \frac{1}{k_{X-1}}
\end{array}\right],
\end{aligned}
$$

$$
\begin{aligned}
& \boldsymbol{A}_{00}^{\prime}=\operatorname{diag}\left[\begin{array}{llll}
-1 / u_{0} & -1 / u_{1} & \ldots & -1 / u_{X-1}
\end{array}\right]+\boldsymbol{A}_{01}^{\prime} \\
& =-\left[\begin{array}{ccc}
\frac{v_{0}}{u_{0}} \frac{1}{k_{0}}+\frac{1}{u_{0}} & & \\
\frac{v_{1}}{u_{1}} \frac{1}{k_{1}} & \frac{v_{1}}{u_{1}} \frac{1}{k_{1}}+\frac{1}{u_{1}} & \\
\vdots & \vdots & \frac{v_{X-1}}{u_{X-1}} \frac{1}{k_{X-1}}+\frac{1}{u_{X-1}} \\
\frac{v_{X-1}}{u_{X-1}} \frac{1}{k_{X-1}} & \cdots & \mathrm{d})
\end{array}\right]
\end{aligned}
$$

\section{REFERENCES}

[1] C. R. Gliech and A. J. Holland, "Keeping track of time: the fundamentals of cellular clocks," Journal of Cell Biology, vol. 219, no. 11, 2020.

[2] J. Gerardin, N. R. Reddy, and W. A. Lim, "The design principles of biochemical timers: circuits that discriminate between transient and sustained stimulation," Cell systems, vol. 9, no. 3, pp. 297-308, 2019.

[3] J. Qian, L. Gelens, and M. Bollen, "Coordination of timers and sensors in cell signaling," BioEssays, vol. 41, no. 3, p. 1800217, 2019.

[4] M. Zhu, W. Chen, V. Mirabet, L. Hong, S. Bovio, S. Strauss, E. M. Schwarz, S. Tsugawa, Z. Wang, R. S. Smith, et al., "Robust organ size requires robust timing of initiation orchestrated by focused auxin and cytokinin signalling," Nature Plants, pp. 1-13, 2020.

[5] A. T. Winfree, The geometry of biological time, vol. 12. Springer Science \& Business Media, 2001.

[6] L. Rensing, U. Meyer-Grahle, and P. Ruoff, "Biological timing and the clock metaphor: oscillatory and hourglass mechanisms," Chronobiology international, vol. 18, pp. 329-369, 2001.

[7] J. J. Dennehy and I.-N. Wang, "Factors influencing lysis time stochasticity in bacteriophage $\lambda$," BMC Microbiology, vol. 11, p. 174, 2011.

[8] R. White, S. Chiba, T. Pang, J. S. Dewey, C. G. Savva, A. Holzenburg, K. Pogliano, and R. Young, "Holin triggering in real time," Proceedings of the National Academy of Sciences, vol. 108, pp. 798-803, 2011.

[9] S. Kannoly, T. Gao, S. Dey, N. Wang, A. Singh, and J. J. Dennehy, "Optimum threshold minimizes noise in timing of intracellular events," iScience, vol. 23, p. 101186, 2020.

[10] C. Schwarz, A. Johnson, M. Kõivomägi, E. Zatulovskiy, C. J. Kravitz, A. Doncic, and J. M. Skotheim, "A precise cdk activity threshold determines passage through the restriction point," Molecular cell, vol. 69, no. 2, pp. 253-264, 2018.

[11] Y. Goldschmidt, E. Yurkovsky, A. Reif, R. Rosner, A. Akiva, and I. Nachman, "Control of relative timing and stoichiometry by a master regulator," PLOS ONE, vol. 10, pp. 1-14, 2015.

[12] I. Nachman, A. Regev, and S. Ramanathan, "Dissecting timing variability in yeast meiosis," Cell, vol. 131, pp. 544-556, 2007.

[13] E. Yurkovsky and I. Nachman, "Event timing at the single-cell level," Briefings in Functional Genomics, vol. 12, pp. 90-98, 2013.

[14] K. Carniol, P. Eichenberger, and R. Losick, "A threshold mechanism governing activation of the developmental regulatory protein $\sigma \mathrm{f}$ in bacillus subtilis," Journal of Biological Chemistry, vol. 279, pp. 14860-14870, 2004.

[15] S. L. Spencer, S. Gaudet, J. G. Albeck, J. M. Burke, and P. K. Sorger, "Non-genetic origins of cell-to-cell variability in TRAILinduced apoptosis," Nature, vol. 459, pp. 428-432, 2009. 
[16] J. Roux, M. Hafner, S. Bandara, J. J. Sims, H. Hudson, D. Chai, and P. K. Sorger, "Fractional killing arises from cell-to-cell variability in overcoming a caspase activity threshold," Molecular Systems Biology, vol. 11, p. 803,2015

[17] A. L. Paek, J. C. Liu, A. Loewer, W. C. Forrester, and G. Lahav, "Cell-to-cell variation in p53 dynamics leads to fractional killing," Cell, vol. 165, no. 3, pp. 631-642, 2016

[18] L. Cai and N. F. X. S. Xie, "Stochastic protein expression in individual cells at the single molecule level," Nature, vol. 440, pp. 358-362, Sept 2006.

[19] J. M. Raser and E. K. O'Shea, "Noise in gene expression: Origins, consequences, and control," Science, vol. 309, pp. 2010 - 2013, 2005

[20] A. Raj and A. van Oudenaarden, "Nature, nurture, or chance: stochastic gene expression and its consequences," Cell, vol. 135, pp. 216-226, 2008.

[21] M. Kærn, T. C. Elston, W. J. Blake, and J. J. Collins, "Stochasticity in gene expression: from theories to phenotypes," Nature Reviews Genetics, vol. 6, pp. 451-464, 2005.

[22] A. M. Corrigan, E. Tunnacliffe, D. Cannon, and J. R. Chubb, "A continuum model of transcriptional bursting," eLife, vol. 5, p. e13051, 2016.

[23] R. D. Dar, B. S. Razooky, A. Singh, T. V. Trimeloni, J. M. McCollum, C. D. Cox, M. L. Simpson, and L. S. Weinberger, "Transcriptional burst frequency and burst size are equally modulated across the human genome," Proceedings of the National Academy of Sciences, vol. 109 pp. 17454-17459, 2012

[24] K. R. Ghusinga, C. A. Vargas-Garcia, and A. Singh, "A mechanistic stochastic framework for regulating bacterial cell division," Scientific Reports, p. 30229, 2016.

[25] P.-Y. Ho, B. M. Martins, and A. Amir, "A mechanistic model of the regulation of division timing by the circadian clock in cyanobacteria," Biophysical Journal, vol. 118, no. 12, pp. 2905-2913, 2020.

[26] K. Rijal, A. Prasad, and D. Das, "Protein hourglass: Exact first passage time distributions for protein thresholds," Phys. Rev. E, vol. 102 p. 052413, Nov 2020 .

[27] A. Singh and J. J. Dennehy, "Stochastic holin expression can account for lysis time variation in the bacteriophage $\lambda$," Journal of the Royal Society Interface, vol. 11, p. 20140140, 2014.

[28] S. Dey, S. Kannoly, P. Bokes, J. Dennehy, and A. Singh, "The role of incoherent feedforward circuits in regulating precision of event timing," bioRxiv, 2020

[29] S. Gupta, S. Fancher, H. C. Korswagen, and A. Mugler, "Temporal precision of molecular events with regulation and feedback," Physical Review E, vol. 101, no. 6, p. 062420, 2020.

[30] K. Biswas and A. Ghosh, "First passage time in post-transcriptional regulation by multiple small rnas," The European Physical Journal E, vol. 44 , no. 2 , pp. $1-10,2021$.

[31] K. R. Ghusinga and A. Singh, "Theoretical predictions on the firstpassage time for a gene expression model," in 2015 54th IEEE
Conference on Decision and Control (CDC), pp. 3864-3869, IEEE, 2015.

[32] K. R. Ghusinga, J. J. Dennehy, and A. Singh, "First-passage time approach to controlling noise in the timing of intracellular events," Proceedings of the National Academy of Sciences, vol. 114, pp. 693698, 2017.

[33] S. Gupta, J. Varennes, H. C. Korswagen, and A. Mugler, "Temporal precision of regulated gene expression," PLoS computational biology, vol. 14, p. e1006201, 2018.

[34] R. Murugan and G. Kreiman, "On the minimization of fluctuations in the response times of autoregulatory gene networks," Biophysical journal, vol. 101, pp. 1297-1306, 2011.

[35] M. C. Lagomarsino, M. Caselle, M. Osella, et al., "Stochastic timing in gene expression for simple regulatory strategies," Nucleic Acids Research, p. gkw1235, 2016.

[36] K. R. Ghusinga and A. Singh, "Effect of gene-expression bursts on stochastic timing of cellular events," in 2017 American Control Conference (ACC), pp. 2118-2123, IEEE, 2017.

[37] K. R. Ghusinga and A. Singh, "Controlling event timing precision for gene expression with external disturbances," in 2020 59th IEEE Conference on Decision and Control (CDC), pp. 5867-5872, 2020.

[38] D. M. Suter, N. Molina, D. Gatfield, K. Schneider, U. Schibler, and F. Naef, "Mammalian genes are transcribed with widely different bursting kinetics," Science, vol. 332, pp. 472-474, 2011.

[39] A. Singh, B. Razooky, C. D. Cox, M. L. Simpson, and L. S. Weinberger, "Transcriptional bursting from the HIV-1 promoter is a significant source of stochastic noise in HIV-1 gene expression," Biophysical journal, vol. 98, pp. L32-L34, 2010.

[40] N. Kumar, A. Singh, and R. V. Kulkarni, "Transcriptional bursting in gene expression: analytical results for general stochastic models," PLOS Computational Biology, vol. 11, p. e1004292, 2015.

[41] C. R. Bartman, S. C. Hsu, C. C.-S. Hsiung, A. Raj, and G. A. Blobel, "Enhancer regulation of transcriptional bursting parameters revealed by forced chromatin looping," Molecular Cell, vol. 62, pp. 237-247, 2016

[42] S. Chong, C. Chen, H. Ge, and X. S. Xie, "Mechanism of transcriptional bursting in bacteria," Cell, vol. 158, pp. 314-326, 2014.

[43] T. Fukaya, B. Lim, and M. Levine, "Enhancer control of transcriptional bursting," Cell, vol. 166, pp. 358-368, 2015.

[44] A. Singh, B. S. Razooky, R. D. Dar, and L. S. Weinberger, "Dynamics of protein noise can distinguish between alternate sources of geneexpression variability," Molecular Systems Biology, vol. 8, p. 607, 2012

[45] Z. Vahdat, K. Nienałtowski, Z. Farooq, M. Komorowski, and A. Singh, "Information processing in unregulated and autoregulated gene expression," in 2020 European Control Conference (ECC), pp. 258-263, IEEE, 2020.

[46] T.-T. Lu and S.-H. Shiou, "Inverses of $2 \times 2$ block matrices," Computers \& Mathematics with Applications, vol. 43, pp. 119-129, 2002. 